Proceedings of XIX International Scientific Conference "New Technologies and Achievements in Metallurgy, Material Engineering, Production Engineering and Physics", Częstochowa, Poland, June 7-8, 2018

\title{
Simulation of the Influence of Corneal Thickness on the Value of Intraocular Pressure
}

\author{
M. Sroga, M. Dośpiat* and R. Matusiak \\ Częstochowa University of Technology, Faculty of Production Engineering and Materials Technology, \\ Institute of Physics, al. Armii Krajowej 19, 42-200 Częstochowa, Poland
}

To determine the influence of corneal thickness on intraocular pressure a measurement on biomechanical eye model was build using a CAD-type software. The model assumes an average corneal thickness of $527 \mu \mathrm{m}$ and pressure prevailing in the anterior chamber of the eye equal to $16 \mathrm{~mm} \mathrm{Hg}$. Then, assuming a constant thickness of the cornea, a curve was built depicting changes in the corneal topography due to the action of the air puff for various intraocular pressure values. The pressure ranged from 8 to $24 \mathrm{~mm} \mathrm{Hg}$. Then, for a constant intraocular pressure equal to $16 \mathrm{~mm} \mathrm{Hg}$, the corneal thickness in the model was changed from 400 to 700 , every $25 \mu \mathrm{m}$, and such a modeled eye was subjected to the same air puff. From the comparison of deformations resulting from the change of thickness with changes resulting from different pressures at the average corneal thickness, the range of distortion of the intraocular pressure measurement was obtained, depending on the thickness of the cornea.

DOI: 10.12693/APhysPolA.135.166

PACS/topics: anatomy of eye, mechanical properties of tissues and organs, biomechanics

\section{Introduction}

Glaucoma along with retinopathy and AMD is one of the leading causes of blindness in developed countries [1]. Measurement of intraocular pressure plays an important role in the study of people at risk of glaucoma. Raised intraocular pressure (above $20 \mathrm{~mm} \mathrm{Hg}$ ) is one of the factors indicating an increased risk of this disease [2]. That is why ongoing research on the implementation and development of intraocular pressure (IOP) screening methods that will be able to conduct various professional groups related to the protection of vision. The limitation of previously used methods was their invasiveness, while the non-invasive air puff method was characterized by considerable inaccuracy. One of the most important factors significantly affecting the incorrect reading of the intraocular pressure measurement is the thickness of the cornea. Currently, we distinguish two trends of leveling the false results resulting from its different thickness. The first method is based on the analysis of the mechanical hysteresis of the deformation of its surface as a result of the measurement [3]. The second method involves the measurement of corneal thickness (pachymetry) and the development of numerical dependences of the measurement deviation depending on its thickness [4].

The study analyzed the biomechanical model of the eye built in the CAD environment in order to determine the effect of various corneal thickness on the reading of the pressure measurement at given constant conditions of intraocular pressure in the model eye.

*corresponding author; e-mail: mdospial@wp.pl

\section{Research methodology and material}

The research used the SOLIDWORKS 2016 (Dassault Systèmes) program, which is a CAD type program used to create models, and then on the basis of performance of executive or assembly drawings. In this program, a simplified three-dimensional model of the human eye (with a spherical cornea) was created, which served as a base for further numerical analysis.

The SOLIDWORKS Simulation addon used in it was used to analyze the FEA's pressure acting on the front part of the cornea.

\subsection{Model geometry}

Figure 1 presents a cross-section through the eye model, which was used for research in the presented work. Corneal curvatures were taken from the literature [5], while the curvature of the sclera was adjusted, so that the total length of the eyeball was $24 \mathrm{~mm}$.

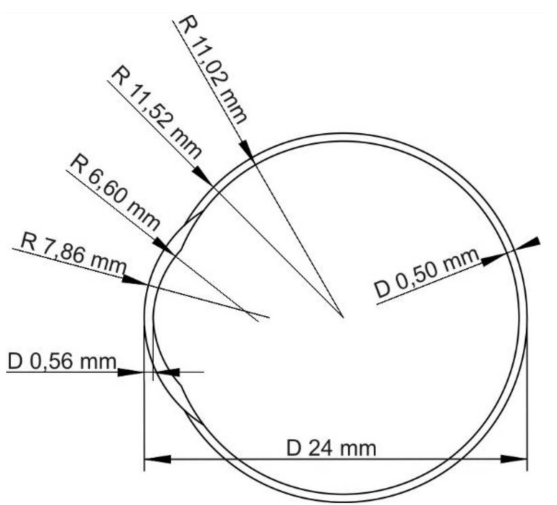

Fig. 1. The cross-section of eye model with the curvature parameters. 


\subsection{Material properties}

In the model, the materials of all structures were presented as linear and isotropic in order to simplify the simulation. For each of them, Young's module, Poisson's number and density were taken into account. The parameters of all structures are summarized in Table I.

TABLE I

The comparison of materials properties of selected structures used in the simulation [6].

\begin{tabular}{c|c|c|c}
\hline \hline Structure & $\begin{array}{c}\text { Young's module } \\
{[\mathrm{MPa}]}\end{array}$ & $\begin{array}{c}\text { Poisson's } \\
\text { number }\end{array}$ & Density \\
\hline cornea & 0.4 & 0.42 & 1060 \\
sclera & 3.0 & 0.47 & 1076
\end{tabular}

\subsection{Simulation parameters}

Simulation of the air puff tonometer was performed by applying an air stream, with a value of $80 \mathrm{~mm} \mathrm{Hg}$, orthogonally to the anterior surface of the cornea (Fig. 2). The test pressure value has been determined relative to the study included in publications [7]. The central corneal thickness was changed to 400 to $700 \mu \mathrm{m}$ in $25 \mu \mathrm{m}$ increments, and the thickness of $526 \mu \mathrm{m}$ was taken as the reference point, taking into account the studies presented in the publication [8].
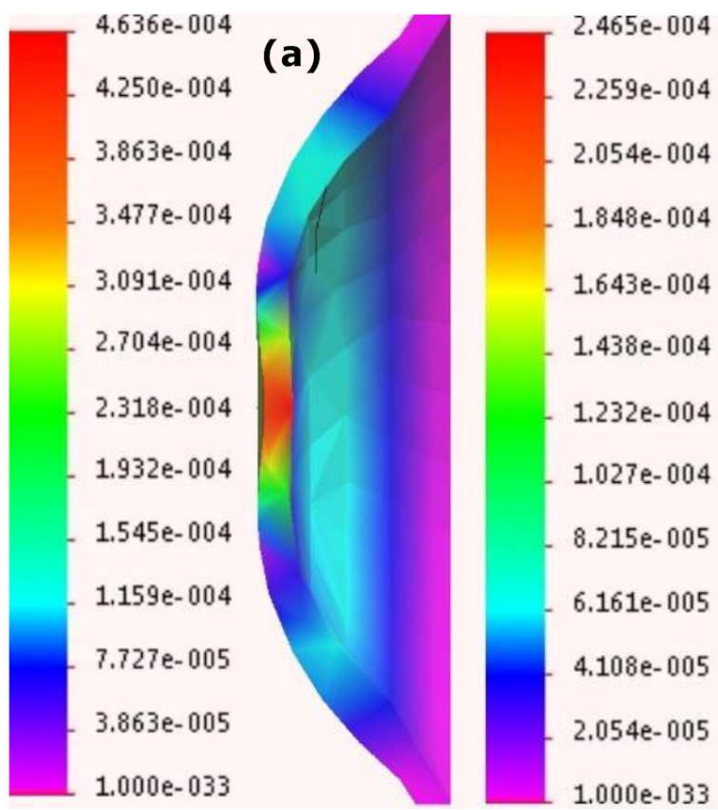

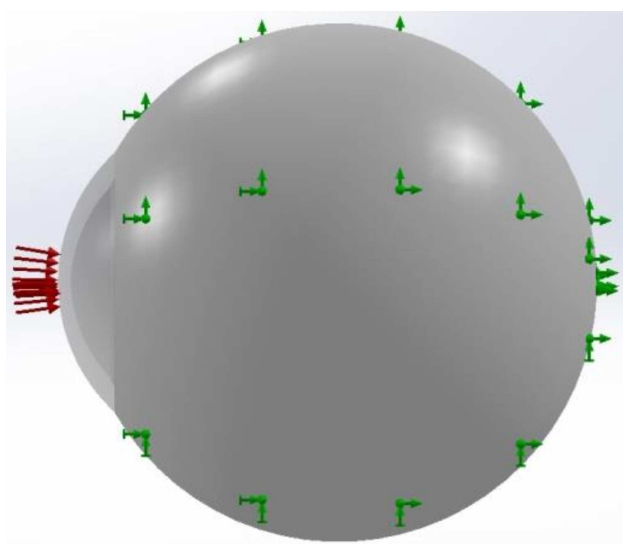

Fig. 2. Graphics showing simulation's parameters.

\section{Results and their discussion}

Figure 3 presents exemplary results of deformation of the corneal front under the influence of the air stream, obtained for the simulation parameters: intraocular pressure $16 \mathrm{~mm} \mathrm{Hg}$, stream pressure $80 \mathrm{~mm} \mathrm{Hg}$, and corneal thickness respectively: $400 \mu \mathrm{m}$ (Fig. 3a), $560 \mu \mathrm{m}$ (Fig. 3b), $700 \mu \mathrm{m}$ (Fig. 3c). On the basis of the obtained results, the eye sections were analyzed to determine changes in the corneal curvature for different values of its thickness and constant pressure inside the eye. The results obtained are shown in Fig. 4.
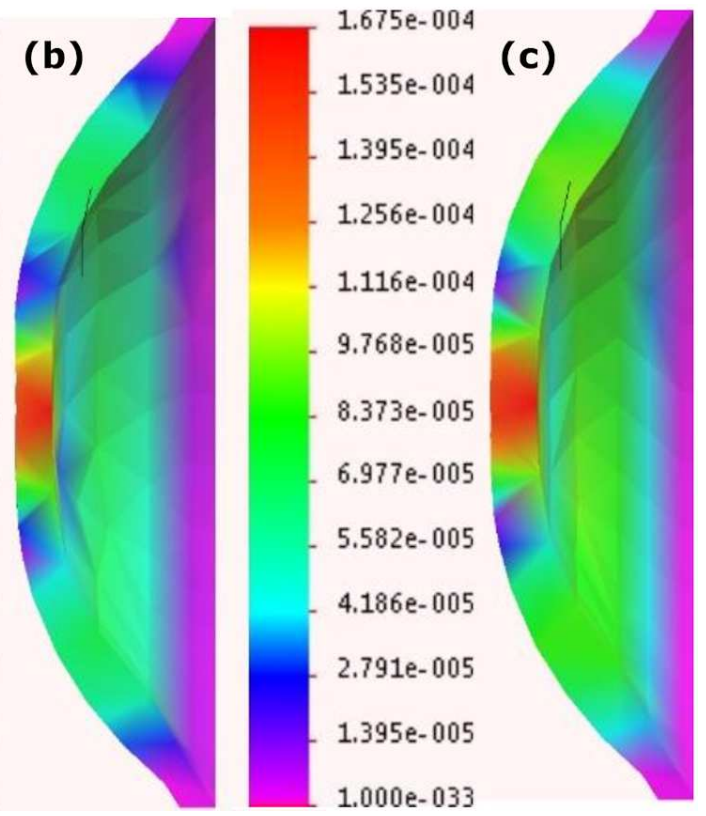

Fig. 3. Simulation results, for the total strain (URES) of the corneal model for the central thickness: (a) $400 \mu \mathrm{m}$, (b) $560 \mu \mathrm{m}$, (c) $700 \mu \mathrm{m}$.

In the next stage of the study, the influence of intraocular pressure on the change of curvature of the cornea was analyzed, assuming a constant thickness of $526 \mu \mathrm{m}$. As in the previous case, the pressure of the air stream used was $80 \mathrm{~mm} \mathrm{Hg}$. In turn, the intraocular pressure parameter in subsequent simulations changed from 8 to $24 \mathrm{~mm} \mathrm{Hg}$, with a step of $2 \mathrm{~mm} \mathrm{Hg}$. The obtained results of the analysis of corneal curvature changes at variable pressure were presented in Fig. 5. 


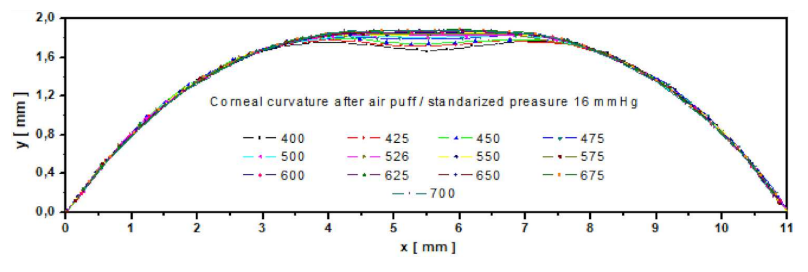

Fig. 4. The deformation simulation results for different thickness of the cornea (in $\mu \mathrm{m}$ ), for constant intraocular pressure.

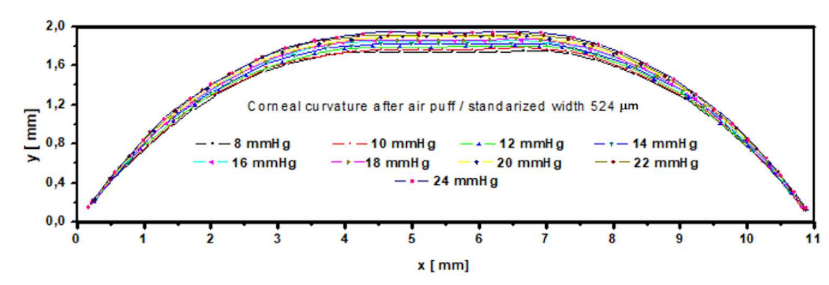

Fig. 5. The deformation simulation results for different intraocular pressures, for constant thickness of the cornea.

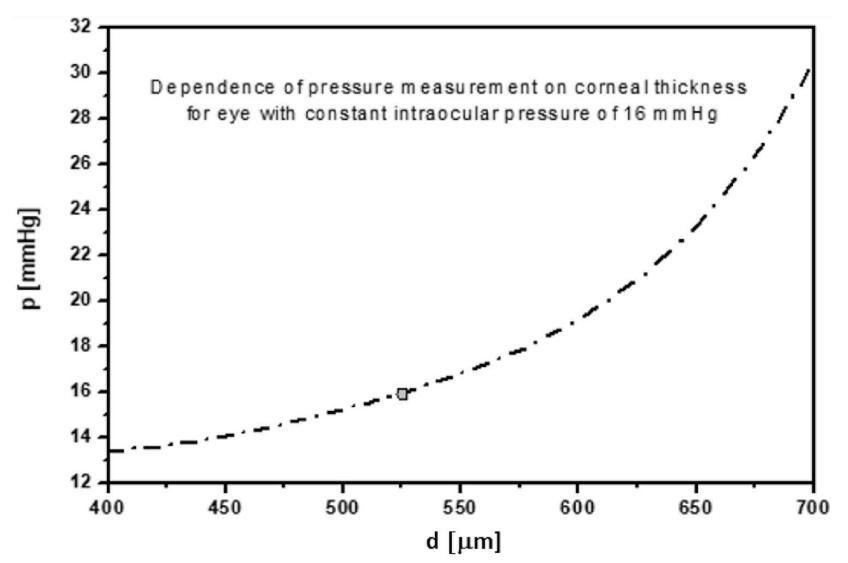

Fig. 6. The dependence of pressure measurement on corneal thickness for eye with constant intraocular pressure of $16 \mathrm{~mm} \mathrm{Hg}$.

Based on the analysis of changes in the topography of the cornea, its apex was numerically fitted (using polynomials of various degrees) describing changes in flattening, depending on corneal thickness or intraocular pressure. Comparison of the obtained results allowed to create a model of the intraocular pressure reading error depending on the thickness of the cornea for IOP equal to $16 \mathrm{~mm} \mathrm{Hg}$. The function of the dependence of reading error on corneal thickness is shown in Fig. 6. A gray point on the obtained function indicates the correct pressure reading which corresponded to the model assumptions.

\section{Conclusion}

The biomechanical model of the eye was made at work. The corneal structure was simplified and assumed a spherical structure. The developed model allowed the analysis of changes in the corneal curvature caused by the action of the air stream as in the case of the application of the intraocular pressure measurement method of the air puff type.

The analysis of changes in the position of the corneal peak under the influence of the air stream for different values of intraocular pressure and different corneal thickness, after comparing these values, allowed to determine the numerical dependence of error reading pressure measurement caused by non-standard corneal thickness. Based on the obtained dependence, the size of the correction of the intraocular pressure for the measurement of it by the air puff method can be determined and the result obtained to the standard in the field of qualification among people at risk of glaucoma. The changes obtained can be used in the context of both theoretical and practical research on corneal biomechanics. However, in further studies, it seems reasonable to introduce selected improvements to the model, e.g. within the eye structure (taking into account more structures and their advanced topography) and analysis of internal flows in the anterior and posterior chambers, which allow to obtain more accurate results of intraocular pressure deviations.

\section{References}

[1] D. Szmurło, T. Fundament, G. Kopeć, D. Brzyski, M. Władysiuk, K. Łanda, Access to ophthalmic care in Poland, Report of the Central and Eastern European Society of Technology Assessment in Health Care, developed in cooperation with the Watch Health Care Foundation of the Professor Jacek Ruszkowski name, 2012.

[2] S. Yoshihiko, Surv. Ophthalmol. 34, 413 (1990).

[3] M. Iester, M. Mete, M. Figus, P. Frezzotti, J. Cataract Refract. Surg. 35, 1623 (2009).

[4] T. Huseynova, G.O. Waring IV, C. Roberts, R.R. Krueger, M. Tomita, Am. J. Ophthalmol. 157, 885 (2014).

[5] J. Nishiyama, Imin Kao, M. Kaneko, in: Proc. 10th Int. Conf. on Ubiquitous Robots and Ambient Intelligence, Jeju (South Korea), 2013, p. 134.

[6] Peishan Dai, Yali Zhao, Hanwei Sheng, Ling Li, Jing Wu, J. Mech. Med. Biol. 17, 1750038 (2017).

[7] Y. Kurita, Y. Iida, R. Kempf, M. Kaneko, in: Proc. Int. Conf. on Information Acquisition, Hong Kong (China), 2005, p. 338.

[8] J. Vroon, J.H. de Jong, A. Aboulatta, A. Eliasy, F.C.T. van der Helm, J.C. van Meurs, D. Wong, A. Elsheikh, Biomech. Model. Mechanobiol. 17, 2 (2018). 